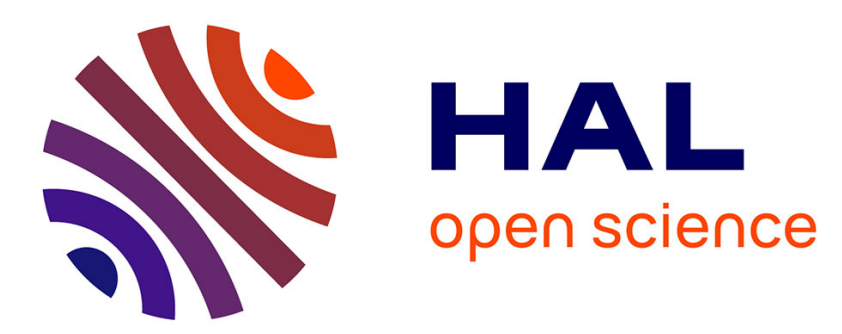

\title{
Vivre avec un handicap psychique à domicile: une approche statistique par les arrangements pratiques
}

\author{
Jean-Sébastien Eideliman
}

\section{To cite this version:}

Jean-Sébastien Eideliman. Vivre avec un handicap psychique à domicile: une approche statistique par les arrangements pratiques. Revue française des affaires sociales, 2009, Handicap psychique et vie quotidienne, 1 (1-2), pp.41-63. hal-01241826

\section{HAL Id: hal-01241826 \\ https://hal.univ-lille.fr/hal-01241826}

Submitted on 11 Dec 2015

HAL is a multi-disciplinary open access archive for the deposit and dissemination of scientific research documents, whether they are published or not. The documents may come from teaching and research institutions in France or abroad, or from public or private research centers.
L'archive ouverte pluridisciplinaire HAL, est destinée au dépôt et à la diffusion de documents scientifiques de niveau recherche, publiés ou non, émanant des établissements d'enseignement et de recherche français ou étrangers, des laboratoires publics ou privés. 


\title{
Vivre avec un handicap psychique à domicile : une approche statistique par les arrangements pratiques
}

\author{
Jean-Sébastien Eideliman*
}

\section{- Introduction}

Suite à la loi sur «l'égalité des droits et des chances, la participation et la citoyenneté des personnes handicapées » de 2005, les Commissions des droits et de l'autonomie des personnes handicapées (CDAPH) sont chargées de reconnaître et de compenser le handicap psychique de toute personne qui en fait la demande ${ }^{1}$. Cette institutionnalisation de la catégorie de handicap psychique, demandée depuis longtemps par des associations comme l'UNAFAM (Union nationale des amis et familles de malades psychiques), laisse cependant ouverte la question de la définition précise de la catégorie et des moyens de recenser les porteurs de ces nouveaux handicaps. Le rapport Charzat (2002), qui a largement inspiré cette entrée du handicap psychique dans la loi, relevait diverses causes possibles (troubles dépressifs graves, états psychotiques ou névrotiques, états limites, détérioration mentale liée à l'âge, à des intoxications ou à des affections neurologiques) et diverses déficiences à l'origine potentielle d'un handicap psychique (voir encadré 1). Il insistait sur la mauvaise prise en compte des conséquences sociales des troubles psychiques, en particulier la stigmatisation qui en résulte, la souffrance pour les personnes concernées et leur entourage, la fragilité et l'isolement qui les accompagne, ou encore le poids des traitements habituellement mis en place.

\footnotetext{
* Maître de conférences en sociologie à l'université Lille 3 et membre du CeRIES (Centre de recherche «Individus, Épreuves, Sociétés»- Lille 3).

1. Cet article a été préparé avec la collaboration de Florence Weber, que je remercie ici, ainsi que Séverine Gojard, à qui les notions développées dans cet article doivent beaucoup.
} 


\section{Encadré 1 : Les différentes dimensions des déficiences psychiques}

Troubles de la volition (impossibilité d'agir par incapacité à vouloir et décider, négativisme, ou au contraire incapacité à s'empêcher d'agir, compulsions obsessionnelles).

Troubles de la pensée (idées obsessionnelles, fuite ou incohérence des idées, lenteur ou appauvrissement de la pensée, délire).

Troubles de la perception (hallucinations, déréalisation).

Troubles de la communication et du langage, repli autistique.

Troubles du comportement (agitation, agressivité contre soi et contre les autres, rites obsessionnels, phobies).

Troubles de l'humeur (troubles dépressifs ou états maniaques, c'est-à-dire états d'excitation et d'agitation psychomotrice).

Troubles de la conscience et de la vigilance.

Troubles intellectuels (difficultés de conceptualisation et d'abstraction, troubles de la mémoire, de l'attention, du jugement, de l'orientation temporelle et spatiale).

Troubles de la vie émotionnelle et affective (anxiété, angoisse, indifférence, discordance ou instabilité affective, troubles du caractère).

Expressions somatiques (somatisations, plaintes, altérations de l'état général).

Source : Rapport Charzat (2002), Guide barème pour l'évaluation des déficiences et incapacités des personnes handicapées.

Si ce rapport, mettant en avant le besoin d'aide des personnes visées par ce nouveau concept, a pu avoir des effets si rapides, c'est qu'il a été rédigé dans un contexte de bouleversement de l'organisation du secteur psychiatrique et plus largement du champ de prise en charge des personnes handicapées ou dépendantes. Du côté de la psychiatrie, le mouvement de fond de désinstitutionnalisation et de développement de prises en charge ambulatoires (Ehrenberg et Lovell, 2001 ; Velpry, 2008) se poursuit, alors qu'un élargissement des perspectives et des publics visés est de plus en plus à l'ordre du jour, à travers la promotion de la «santé mentale», qui a vocation à dépasser l'approche strictement psychiatrique des troubles mentaux pour englober les aspects médico-sociaux de la prise en charge des désordres mentaux, petits ou grands. Si la naissance officielle du handicap psychique s'inscrit bien dans un tel paysage puisque cette nouvelle notion vise à saisir les diverses conséquences sociales d'un large ensemble de problèmes psychiques, elle subit cependant de plein fouet le développement de l'incertitude nosographique en psychiatrie au sein de laquelle les débats classificatoires font rage (Ehrenberg et Lovell, 2001b; Wilson, 1993). C'est en partie parce que les professionnels du secteur psychiatrique ne sont pas tous persuadés, loin s'en faut, que l'optique du handicap est légitime en matière de troubles mentaux, 
que la notion de handicap psychique a mis longtemps à émerger. Pour certains, traiter les conséquences des troubles psychiques sans soigner le mal à la racine n'a pas de sens et conduit à figer des problèmes par nature évolutifs (Libermann, 1988).

Dans ce contexte, déterminer qui sont les personnes atteintes de handicap psychique est difficile. Le réflexe le plus fréquent consiste à prendre à rebours le processus qui va de la maladie à sa prise en charge, en prenant comme indicateur de l'existence de troubles le fait qu'une prise en charge psychiatrique a été mise en place. Ainsi, les données sont nombreuses sur les personnes fréquentant des établissements psychiatriques (Chapireau, 2003 et 2004) ou, si l'on souhaite cibler les personnes vivant en domicile ordinaire, ayant un suivi psychique régulier (Anguis, Chapireau et De Peretti, 2003; Cases et Salines, 2004). Certains auteurs choisissent d'autres indicateurs, comme par exemple la mise sous tutelle pour raisons psychiques (Séraphin, 2003), qui ont en commun d'être des indicateurs produits à partir de catégories administratives ou médicales, et ont de ce fait l'avantage de donner lieu à des comptages statistiques aisément consultables.

À l'inverse, l'enquête Handicaps-Incapacités-Dépendance (HID) menée par l'INSEE en institutions (vagues de l'enquête passées en 1998 et en 2000) et en ménages ordinaires (vagues 1999 et 2001) a la particularité de ne pas présupposer de définition des troubles et de laisser les individus interrogés déclarer eux-mêmes leurs problèmes de santé, qu'ils soient physiques, sensoriels ou mentaux ${ }^{1}$ (voir encadré 2 ). En partant de cette enquête, qui livre à l'analyse un large échantillon de personnes en proie aux difficultés quotidiennes provenant de problèmes de santé les plus divers, il est ainsi possible de chercher à définir le handicap psychique à partir de ses manifestations les plus concrètes et de porter en retour un regard critique sur les catégories médico-administratives et les indicateurs (comme par exemple l'existence d'un suivi psychique régulier) qui servent le plus souvent à le circonscrire.

Nous avons déjà eu l'occasion d'exploiter de la sorte cette enquête, alors que nous nous posions des questions sur les modalités de prise en charge des personnes handicapées et dépendantes (Eideliman et Gojard, 2008). Ces interrogations, qui provenaient d'enquêtes ethnographiques portant sur la mobilisation (ou l'absence de mobilisation) d'aidants familiaux et professionnels autour de personnes dépendantes (Weber, Gojard et Gramain, 2003), ont donné lieu à la formulation de la notion d'arrangements pratiques ${ }^{2}$, qui vise à décrire l'ensemble des modalités concrètes d'organisation de la vie quotidienne qui permettent de surmonter des problèmes de santé handicapants. L'objectif de cet article est de faire fonctionner de nouveau cette notion pour comprendre qui sont les personnes susceptibles d'être visées

1. À la restriction près que les personnes n'étant pas en mesure de répondre elles-mêmes au questionnaire ont été « remplacées » dans cette tâche par un membre de leur entourage, ce que les données précisent heureusement systématiquement.

2. Au sens des «arrangements privés» (Pennec, 2005) ou encore des «arrangements de famille» (Bessière, 2004). 
par la notion de handicap psychique et mettre à l'épreuve les indicateurs habituellement utilisés. Après avoir examiné en détail les liens entre handicap psychique et suivi psychique, nous proposerons une autre manière de décrire les différentes configurations dans lesquelles sont prises les personnes ayant au quotidien des difficultés d'ordre psychique.

\title{
Encadré 2 : Présentation des enquêtes Handicaps-Incapacités- Dépendance (HID)
}

\begin{abstract}
Préparé et réalisé par un groupe de projet constitué autour de l'Institut national de la statistique et des études économiques (Insee) (Letourmy, 1998), le dispositif d'enquête HID est composé de plusieurs vagues de recueil de données, qui se sont déroulées de 1998 à 2001, en institutions et en domiciles ordinaires. Nous utilisons, dans cet article, la deuxième de ces enquêtes, menée en 1999, auprès de 20000 individus vivant à domicile et sélectionnés à partir de l'enquête "Vie quotidienne et santé " (VQS), complémentaire du recensement de la population.

Intervenant à une période où l'étanchéité entre les politiques publiques du handicap (pour les actifs) et de la dépendance (pour les personnes âgées) commence à être contestée, l'enquête vise à réunir des données, jusque-là éparses, sur ces deux populations (Ravaud, Letourmy et Ville, 2002). Pour ce faire, elle interroge des individus de tous âges, répartis sur l'ensemble du territoire national, en surreprésentant ceux qui déclarent rencontrer des difficultés dans leur vie quotidienne.
\end{abstract}

\section{- Handicap psychique rime-t-il avec suivi psychique?}

La notion de handicap psychique pose à ceux qui veulent s'en saisir le problème de sa définition précise, tant elle a plus été posée comme un principe que comme une situation précisément décrite. En choisissant de l'approcher à travers les données de l'enquête HID de 1999, on ne se facilite a priori pas la tâche puisque cette enquête a été construite et conduite à une période où la catégorie de handicap psychique n'existait pas en tant que telle et ne donnait donc pas lieu à l'élaboration de questions spécifiques pour la cibler. Lorsque l'on cherche cependant à interroger cette nouvelle catégorie et à en cerner les contours, le défaut que l'on vient de souligner peut rapidement se muer en qualité, dans la mesure où la multitude de questions présentes dans l'enquête HID sur les activités de la vie quotidienne peuvent permettre de reconstruire, facette après facette, ce qui s'apparente à l'actuelle catégorie de handicap psychique, plutôt que de faire confiance à un unique indicateur censé exprimer la totalité du processus complexe dont résulte le handicap psychique. 


\section{Le handicap psychique à travers ses manifestations quotidiennes}

L'enquête HID reprend dans sa structure les différents niveaux composant le handicap selon le schéma de Wood : les déficiences (niveau lésionnel), les incapacités (niveau fonctionnel) et le désavantage (niveau social) ${ }^{1}$. Suite à une réflexion collective qui a déjà montré sa fécondité (Eideliman et Gojard, 2008), nous avons choisi de partir des incapacités, c'est-à-dire de la manière dont les individus interrogés effectuent (ou non) un certain nombre d'activités de la vie quotidienne, pour cerner les conséquences concrètes et quotidiennes d'un éventuel handicap psychique. Parmi l'ensemble des activités proposées par l'enquête HID de 1999, nous avons retenu sept questions qui dessinent les contours habituellement retenus du handicap psychique (voir encadré 1) :

- En dehors de problèmes liés à la surdité, communiquez-vous avec votre entourage sans l'aide de quelqu'un?

- Avez-vous des difficultés à parler? (Y compris pour raison de bégaiement.)

- Vous arrive-t-il de ne plus vous souvenir à quel moment de la journée on est?

- Avez-vous des difficultés à trouver votre chemin quand vous sortez?

- Vous arrive-t-il, par votre comportement, de vous mettre en danger (risques de se blesser...)?

- Vous reproche-t-on parfois d'être trop agressif ou impulsif?

- Arrive-t-il qu' on critique votre comportement pour d'autres raisons (que l'agressivité ou l'impulsivité)?

À ces questions, les individus interrogés pouvaient répondre par l'affirmative ou la négative, mais aussi signaler qu'ils effectuaient ces activités avec difficultés ou en ayant recours à de l'aide humaine. Après avoir analysé et comparé les réponses à ces différentes questions, nous avons construit trois variables qui permettent d'approcher le handicap psychique à partir des incapacités déclarées ( $c f$. tableaux 1 à 3 ).

Les problèmes de communication concernent principalement les plus jeunes et les plus âgés. Lorsque les problèmes sont importants, le niveau d'aide ${ }^{2}$

1. Cependant, l'enquête intègre également un grand nombre de données environnementales, préfigurant ainsi la classification internationale adoptée en 2001 (CIF - Classification internationale du fonctionnement, du handicap et de la santé).

2. Le niveau d'aide est un indicateur que nous avons eu l'occasion de définir en détail ailleurs (Eideliman et Gojard, 2008). Il distingue des situations où aucune aide humaine n'est déclarée (vie sans aide et sans difficultés, vie sans aide avec des difficultés, vie sans aide avec de grosses difficultés, vie sociale restreinte), d'autres où une aide humaine au moins est mentionnée (aide substituable, aide sociale, aide vitale), enfin des situations où des aides techniques lourdes aboutissent à une situation de confinement à domicile. L'aide substituable correspond à l'intervention d'aidants pour des activités dites substituables, c'est-à-dire qu'on peut ne pas faire en faisant appel à un service professionnel auquel n'importe qui pourrait avoir accès sous réserve d'être solvable (restaurant, pédicure). L'aide sociale correspond à l'intervention d'aidants pour des activités dites sociales, c'est-à-dire qu'on peut ne pas faire au prix d'une restriction de sa vie sociale (sorties, relations avec autrui, etc.). L'aide vitale correspond à l'intervention d'aidants pour des activités dites vitales, c'est-à-dire qu'on ne peut pas ne pas faire, à moins de sortir d'un mode d'existence ordinaire. 
est élevé, avec de nombreuses situations de confinement à domicile et d'aide vitale. Lorsque les problèmes sont plus légers, l'aide vitale est encore fréquente, mais aux côtés de l'aide sociale. Des aidants familiaux sont très souvent mobilisés, mais souvent en association avec des aidants professionnels. Les déficiences les plus fréquemment associées sont des troubles du comportement. Enfin, les personnes qui subissent ces problèmes font partie des catégories les plus populaires de l'échantillon général de l'enquête (qu'on mesure leur niveau social par leur propre PCS, celle de leur père et leurs revenus), mais cela ne les distingue pas des personnes ayant des problèmes d'orientation ou de comportement, qui sont situées au même niveau de l'échelle sociale.

\section{Tableau 1 : Problèmes de communication}

\begin{tabular}{|l|c|c|}
\hline \multicolumn{1}{|c|}{$\begin{array}{c}\text { Problèmes de communication } \\
\text { (y compris difficultés à parler) }\end{array}$} & Fréquence & $\%$ \\
\hline Aucun problème & 15540 & 91,58 \\
Problèmes légers & 821 & 4,84 \\
Problèmes importants & 608 & 3,58 \\
\hline
\end{tabular}

Tableau 2 : Problèmes d'orientation

\begin{tabular}{|l|c|c|}
\hline \multicolumn{1}{|c|}{$\begin{array}{c}\text { Problèmes d'orientation } \\
\text { (spatiale ou temporelle) }\end{array}$} & Fréquence & \% \\
\hline Aucun problème & 14679 & 86,50 \\
Problèmes légers & 1118 & 6,59 \\
Problèmes importants & 1172 & 6,91 \\
\hline
\end{tabular}

\section{Tableau 3 : Problèmes de comportement}

\begin{tabular}{|l|c|c|}
\hline $\begin{array}{c}\text { Problèmes de comportement } \\
\text { (agressivité, mise en danger de soi...) }\end{array}$ & Fréquence & \% \\
\hline Aucun problème & 12378 & 72,94 \\
Problèmes légers autodéclarés & 1995 & 11,76 \\
Problèmes importants autodéclarés & 1729 & 10,19 \\
Problèmes déclarés par autrui & 867 & 5,11 \\
\hline
\end{tabular}

1. Il nous a semblé qu'en matière de troubles du comportement, il était tout à fait différent que l'on mentionne soi-même des troubles ou que quelqu'un d'autre en mentionne pour soi. Comme l'enquête HID indique si la personne concernée par l'enquête a répondu elle-même au questionnaire ou s'est fait remplacer ou aider par un membre de son entourage, nous avons utilisé cette information pour distinguer une modalité supplémentaire par rapport aux autres variables retenues.

Les problèmes d'orientation concernent principalement des personnes âgées, en particulier des femmes, mais aussi un nombre non négligeable de moins de 20 ans. Lorsque les problèmes sont peu importants, les personnes concernées 
vivent souvent seules, au prix d'une restriction de leur vie sociale. Lorsque les problèmes sont importants, elles vivent très souvent avec leurs parents (dans le cas des plus jeunes) ou leurs enfants (dans le cas des plus âgées) et ont un niveau d'aide élevé. Des aidants familiaux sont très souvent mobilisés, fréquemment en association avec des aidants professionnels. Les déficiences les plus régulièrement associées sont mentales et cognitives.

Les problèmes de comportement touchent le plus souvent des personnes d'âge actif vivant en couple (sauf pour les problèmes déclarés par autrui). Lorsque les problèmes sont importants (ou déclarés par autrui), les niveaux d'aide sont encore une fois élevés (confinement à domicile, aide vitale). Les aidants déclarés sont le plus souvent exclusivement familiaux. Les déficiences les plus fréquemment associées sont comportementales et de l'humeur.

Lorsque l'on croise ces divers indicateurs d'incapacités psychiques avec l'existence ou non d'un suivi psychique régulier, on a la surprise de constater que quels que soient le type et le degré des problèmes en cause, le suivi psychique régulier ne concerne jamais plus qu' un quart des personnes affectées. Cette surprise nous a poussé à chercher à en savoir plus sur les déterminants du suivi psychique régulier, pour comprendre quel ensemble de personnes il concerne et dans quelle mesure il peut ou non être considéré comme un indicateur de l'existence d'un handicap psychique.

\section{Les déterminants du suivi psychique}

Sur l'ensemble de l'échantillon de l'enquête HID 1999, 1223 personnes ont déclaré avoir un suivi médical régulier pour des troubles psychiques ou mentaux. Les résultats de la régression logistique présentés au tableau 4 permettent de cerner les effets propres de diverses variables sur la probabilité d'avoir un suivi psychique régulier.

Comme on pouvait s'y attendre, toutes choses égales par ailleurs, plus le handicap est important, plus le suivi psychique est fréquent. De même, les personnes ayant une déficience sur le plan comportemental ont plus de chances d'avoir un suivi psychique régulier. Ces variables ne fournissent pas d'enseignement intéressant en soi, mais servent surtout à contrôler des dimensions dont on sait par avance le rôle, afin de mieux cerner les effets propres des autres variables mobilisées.

Sur le plan socio-économique, il est en revanche surprenant de constater que les situations qui favorisent le recours à un suivi psychique régulier semblent être les cas de déclassement social. En effet, ce sont les personnes ayant à la fois de faibles revenus et une origine sociale élevée qui ont le plus de chances d'avoir un tel suivi. Ce résultat contraste avec les caractéristiques des incapacités psychiques repérées plus haut, qui concernent principalement des personnes d'origine sociale modeste. 
Tableau 4 : Régression logistique. Estimation de la probabilité d'avoir recours à un suivi psychique régulier

\begin{tabular}{|c|c|c|}
\hline Paramètre & Estimation & Khi 2 \\
\hline Intercept & -1.4392 & $<.0001$ \\
\hline FEMME & \multicolumn{2}{|c|}{ référence } \\
\hline homme & -0.3795 & $<.0001$ \\
\hline $0-6$ ans & -0.3223 & 0.1788 \\
\hline $7-20$ ans & -0.2775 & 0.0659 \\
\hline 21-40 ANS & \multicolumn{2}{|c|}{ référence } \\
\hline $41-60$ ans & 0.1554 & 0.1013 \\
\hline 61-80 ans & -1.0160 & $<.0001$ \\
\hline+ de 80 ans & -2.2694 & $<.0001$ \\
\hline vit avec ses parents & 0.1292 & 0.3775 \\
\hline EN COUPLE SANS ENFANT & \multicolumn{2}{|c|}{ référence } \\
\hline en couple avec enfant & -0.2940 & 0.0052 \\
\hline personne seule & 0.4275 & $<.0001$ \\
\hline autre ménage & -0.0558 & 0.6898 \\
\hline$<457$ euros / mois et unité de consommation & 0.3285 & 0.0070 \\
\hline de 458 à 762 euros / mois et UC & 0.4134 & $<.0001$ \\
\hline de 763 à 1219 euros / mois et UC & 0.1486 & 0.1368 \\
\hline PLUS DE 1220 EUROS / MOIS ET / UC & \multicolumn{2}{|c|}{ référence } \\
\hline revenu non connu & 0.0581 & 0.7485 \\
\hline père agriculteur exploitant & -0.5808 & 0.0001 \\
\hline père artisan, commerçant ou chef d'ent. & -0.4160 & 0.0068 \\
\hline PÈRE CADRE OU PIS & \multicolumn{2}{|c|}{ référence } \\
\hline père professions intermédiaires & -0.1945 & 0.2089 \\
\hline père employé & -0.2053 & 0.1712 \\
\hline père ouvrier & -0.4966 & 0.0001 \\
\hline père inactif & -0.5666 & 0.0021 \\
\hline PCS du père inconnue & -0.4431 & 0.0263 \\
\hline VQS : pas de handicap & -0.5198 & 0.0124 \\
\hline VQS : HANDICAP DECLARE & \multicolumn{2}{|c|}{ référence } \\
\hline VQS : handicap objectivé & 0.7089 & $<.0001$ \\
\hline VQS : handicap reconnu & 0.9930 & $<.0001$ \\
\hline Pas d'aidants déclarés & -0.2562 & 0.0023 \\
\hline AIDANTS FAMILIAUX UNIQUEMENT & \multicolumn{2}{|c|}{ référence } \\
\hline Aidants professionnels & 0.2101 & 0.0309 \\
\hline PAS DE DÉFICIENCE COMPORTEMENTALE & \multicolumn{2}{|c|}{ référence } \\
\hline déficience comportementale & 0.6706 & $<.0001$ \\
\hline pas de problèmes de communication & -0.6114 & $<.0001$ \\
\hline PROBLÈMES DE COMMUNICATION & \multicolumn{2}{|c|}{ référence } \\
\hline Gros problèmes de communication & 0.0359 & 0.8067 \\
\hline Pas de problèmes d'orientation & -0.7857 & $<.0001$ \\
\hline PROBLÈMES D'ORIENTATION & \multicolumn{2}{|c|}{ référence } \\
\hline Gros problèmes d'orientation & -0.0362 & 0.7662 \\
\hline Pas de problèmes de comportement & -0.3022 & 0.0043 \\
\hline PROBLÈMES DE COMPORTEMENT & \multicolumn{2}{|c|}{ référence } \\
\hline Gros problèmes de comportement & 0.5041 & $<.0001$ \\
\hline Problèmes de comportement déclarés par autrui & 0.5608 & $<.0001$ \\
\hline
\end{tabular}

Lecture: Pour chaque variable, la modalité de référence apparaît en majuscules. Par rapport à cette modalité, le modèle estime si l'appartenance à telle autre modalité de la même variable augmente ou diminue la probabilité d'avoir un suivi psychique régulier (voir le signe du coefficient de la colonne «estimation»). La dernière colonne indique la marge d'erreur qu'il faut accepter pour que le résultat soit considéré comme significatif. Lorsque la marge d'erreur est inférieure à $5 \%$, on a considéré que le résultat était significatif et la ligne apparaît en gras. Lorsque la marge d'erreur est entre 5 et $10 \%$ (une seule modalité), la ligne apparaît en gras et italique. 
Les personnes d'âge actif ont bien plus de chances d'avoir un suivi psychique régulier que les plus jeunes et les plus âgées. On peut relier ce résultat à deux grands éléments; d'une part, la construction du champ de prise en charge du handicap a depuis son origine partie liée avec la sphère professionnelle, dans la mesure où la reconnaissance d'un handicap, y compris mental, peut donner un statut professionnel particulier ou bien être relié aux conséquences d'un accident du travail (Blanc et Stiker, 1998). D'autre part, les troubles psychiques ont été médicalement construits de telle façon qu'ils apparaissent le plus souvent après l'adolescence mais très rarement au grand âge, où d'autres catégories sont utilisées pour catégoriser d'éventuels problèmes mentaux ou cognitifs. Nous reviendrons en détail sur ce deuxième élément d'explication.

On peut par ailleurs remarquer que prise en charge psychique et prise en charge par des aidants vont de pair : la probabilité d'avoir un suivi psychique régulier est plus faible lorsque aucun aidant n'est déclaré que lorsque des aidants uniquement familiaux sont mentionnés. De façon plus surprenante, la déclaration d'aidants professionnels (seuls ou en complément d'aidants familiaux) augmente cette probabilité par rapport au cas où seuls des aidants familiaux interviennent. Il semble donc que le fait de faire appel à des aidants professionnels (à niveau de handicap donné, rappelons-le) pousse à inclure dans cette prise en charge professionnelle un suivi psychique régulier (à moins que ce ne soit l'inverse).

Enfin, comme on pouvait s'y attendre, le fait d'avoir des problèmes de communication ou d'orientation augmente les chances d'avoir un suivi régulier sur le plan psychique. Mais, comme le laissait prévoir le faible taux de personnes suivies sur le plan psychique, l'augmentation de la sévérité des problèmes de communication ou d'orientation n'engendre pas l'augmentation du recours à un suivi psychique régulier. Il en va autrement pour les problèmes de comportement, pour lesquels le recours à un suivi psychique augmente régulièrement avec l'importance des problèmes.

Il ressort de cet ensemble de résultats d'une part que les personnes ayant un suivi psychique régulier ont un profil différent des personnes ayant des incapacités psychiques puisque les femmes, les personnes d'âge actif et les personnes d'origine sociale élevée y sont bien plus représentées. D'autre part, si incapacités et suivi psychiques sont en partie liés, cette liaison n'est en rien systématique et semble plus robuste pour les problèmes de comportement. Afin de tester plus finement cette liaison, nous allons maintenant reconsidérer la manière dont incapacités et suivi psychique se croisent. 


\section{La nébuleuse des incapacités psychiques}

Nos investigations précédentes nous ont montré qu'incapacités et suivi psychiques étaient certes liés, mais de manière imparfaite et probablement complexe. Le graphique 1 confirme et affine ces résultats en montrant que le recours à un suivi psychique augmente lorsque les incapacités psychiques se cumulent, sans toutefois dépasser des proportions modestes (à peine plus d'un tiers de personnes suivies sur le plan psychique lorsque problèmes de communication, d'orientation et de comportement sont conjointement présents).

Le graphique 1 fait également apparaître à quel point le paysage des incapacités psychiques se présente comme une nébuleuse : il n'y a pas d'associations systématiques entre certaines incapacités mais différentes configurations à peu près aussi fréquentes les unes que les autres.

\section{Graphique 1 : Incapacités psychiques (tous problèmes)}

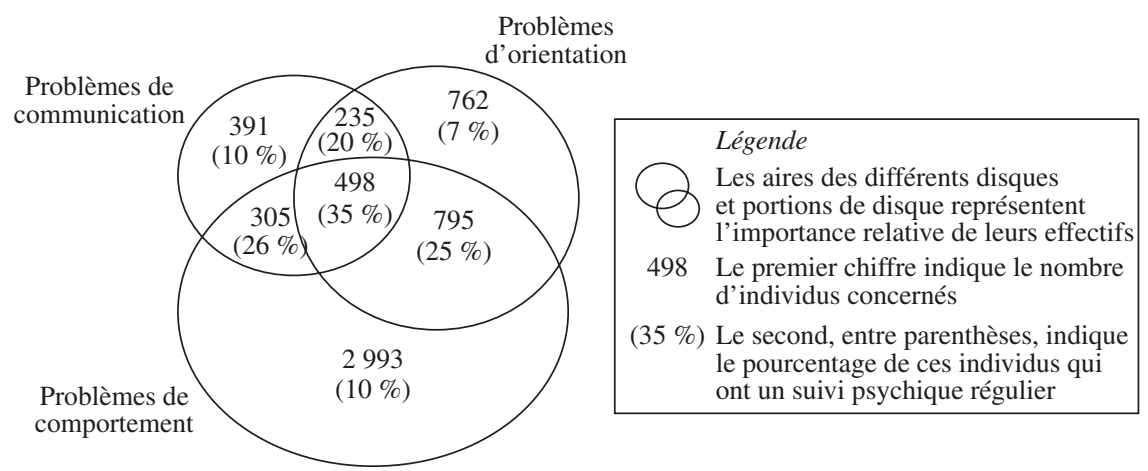

Alors qu' on aurait a priori pu s'attendre à ce que les associations soient plus systématiques pour les personnes ayant des problèmes importants, le graphique 2 montre que les choses sont tout aussi complexes si l'on ne prend en compte que les personnes ayant des problèmes importants de communication, d'orientation et de comportement.

Non seulement la fréquence du recours à un suivi psychique se maintient de façon remarquable à des niveaux semblables à ceux du graphique 1, mais les diverses configurations de cumul (ou de non-cumul) d'incapacités psychiques sont là encore bien réparties en termes d'effectifs, même si les situations où une seule incapacité est présente dominent (surtout pour les problèmes de comportement, bien plus fréquents que les autres dans l'échantillon). 


\section{Graphique 2 : Incapacités psychiques (problèmes importants)}

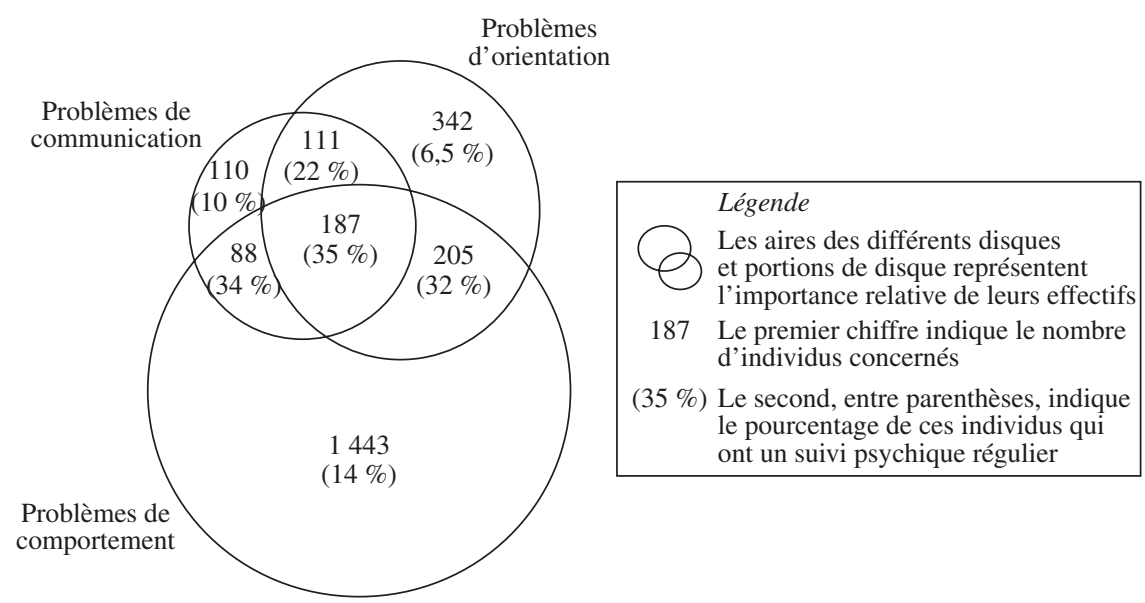

Comment interpréter le fait que la présence d'incapacités psychiques ne donne dans la majorité des cas pas lieu à la mise en place d'un suivi psychique régulier? On pourrait voir dans ce découplement la trace d'un besoin d'aide largement insatisfait : beaucoup de personnes seraient ainsi insuffisamment encadrées par les professionnels du psychisme alors que de véritables limitations découlant de problèmes psychiques les affecteraient au quotidien. La notion de besoin d'aide est centrale dans la mise en place de politiques sociales sur de tels sujets. Comme nous avons déjà eu l'occasion de le développer (Eideliman et Gojard, 2008), l'utilisation de la notion de besoin d'aide pour interpréter des données portant sur l'observation d'activités de la vie quotidienne nous semble cependant poser problème. Sans refaire ici la démonstration, nous rappellerons simplement que le fait que des personnes déclarent avoir des difficultés pour effectuer telle activité du fait de problèmes d'ordre psychique ne signifie pas qu'elles ont besoin, et encore moins envie, d'un suivi psychique régulier. Nous proposons plutôt d'utiliser ces précieuses données sur les activités quotidiennes pour décrire ce que nous avons appelé les «arrangements pratiques» de ces personnes, c'est-à-dire les combinaisons entre divers éléments de leur situation (configuration du ménage, recours éventuels à des aidants, démarches administratives de reconnaissance des difficultés, lieu d'habitation, etc.) qui leur servent à stabiliser une organisation pour se débrouiller au quotidien, faire avec des difficultés plus ou moins limitatives voire, dans certains cas, se passer d'aide humaine ou du recours à des professionnels (y compris du psychisme). Ce sont ces arrangements que nous nous proposons de décrire maintenant, au moyen d'une autre méthode statistique qui leur est particulièrement bien adaptée, l'analyse factorielle. 


\section{- Handicaps psychiques et arrangements pratiques : l'importance du cycle de vie}

Si la régression logistique permet de séparer les influences propres de divers facteurs et par exemple de donner une idée du profil-type des personnes ayant recours à un suivi psychique régulier, l'analyse factorielle est mieux adaptée pour essayer d'y voir clair dans la nébuleuse des incapacités psychiques, que les croisements commentés ci-dessus permettent seulement de deviner (Biland, Eideliman et Gojard, 2008). D'une manière plus générale, l'approche des handicaps par les arrangements pratiques s'accorde particulièrement bien à cette méthode statistique puisqu'il s'agit de mettre en évidence des configurations et des interdépendances, ce que ne permet pas la régression, qui sépare au contraire les influences de chaque variable prise en compte.

Les deux graphiques présentés ci-dessous ${ }^{1}$ sont tirés d'une analyse des correspondances multiples (ACM) qui porte sur l'ensemble des personnes ayant au moins une incapacité psychique. Nous avons en effet choisi d'éliminer de l'échantillon de cette analyse les personnes n'ayant déclaré aucun problème de communication, d'orientation et de comportement, ni aucun suivi psychique régulier, afin de ne pas voir ces personnes ayant de nombreuses caractéristiques communes constituer un pôle saillant dans les résultats de l'ACM, qui nous apprendrait bien peu de choses. L'analyse porte donc sur les 6289 individus ayant déclaré au moins une limitation dans la vie quotidienne liée à des problèmes psychiques, ou bien un suivi psychique régulier. Afin de faciliter la lecture des graphiques 3 et 4, les modalités de certaines variables importantes pour l'interprétation ont été mises en valeur : en gras les modalités du niveau d'aide; en italiques celles des incapacités psychiques; celles des structures de ménage sont soulignées; enfin celles concernant la déclaration d'aidants sont encadrées. Certaines modalités, mal reliées aux axes et situées proches du centre des graphiques, ont été effacées pour assurer une meilleure lisibilité. Toujours pour faciliter la lecture, nous avons ajouté des expressions (en majuscules et en gras) qui permettent de caractériser grossièrement les différents pôles de chaque graphique.

\footnotetext{
$\overline{1 .}$ Comme toute analyse factorielle, l'analyse des correspondances multiples consiste à ramener le nuage de points à un ensemble d'axes factoriels qui se partagent l'inertie (variance) totale du nuage. Le premier axe explique la plus grande partie de cette inertie, le second explique une part plus petite et ainsi de suite. Dans l'analyse présentée ici, les deux premiers axes se distinguent des suivants : le premier explique 11,12\% de l'inertie totale et le second 8,04\%, alors que les suivants sont en dessous de $5,7 \%$. Ces valeurs peuvent paraître faibles, mais elles découlent mécaniquement du grand nombre de modalités actives introduites dans l'analyse et ne signalent pas une moindre valeur de l'analyse. Nous avons choisi de commenter également le troisième axe qui, s'il explique un peu moins de 5,7\% de l'inertie totale, reste significatif et permet d'apporter nuances et précisions.
} 
Graphique 3 : Handicaps psychiques et arrangements pratiques - Axes 1 et 2

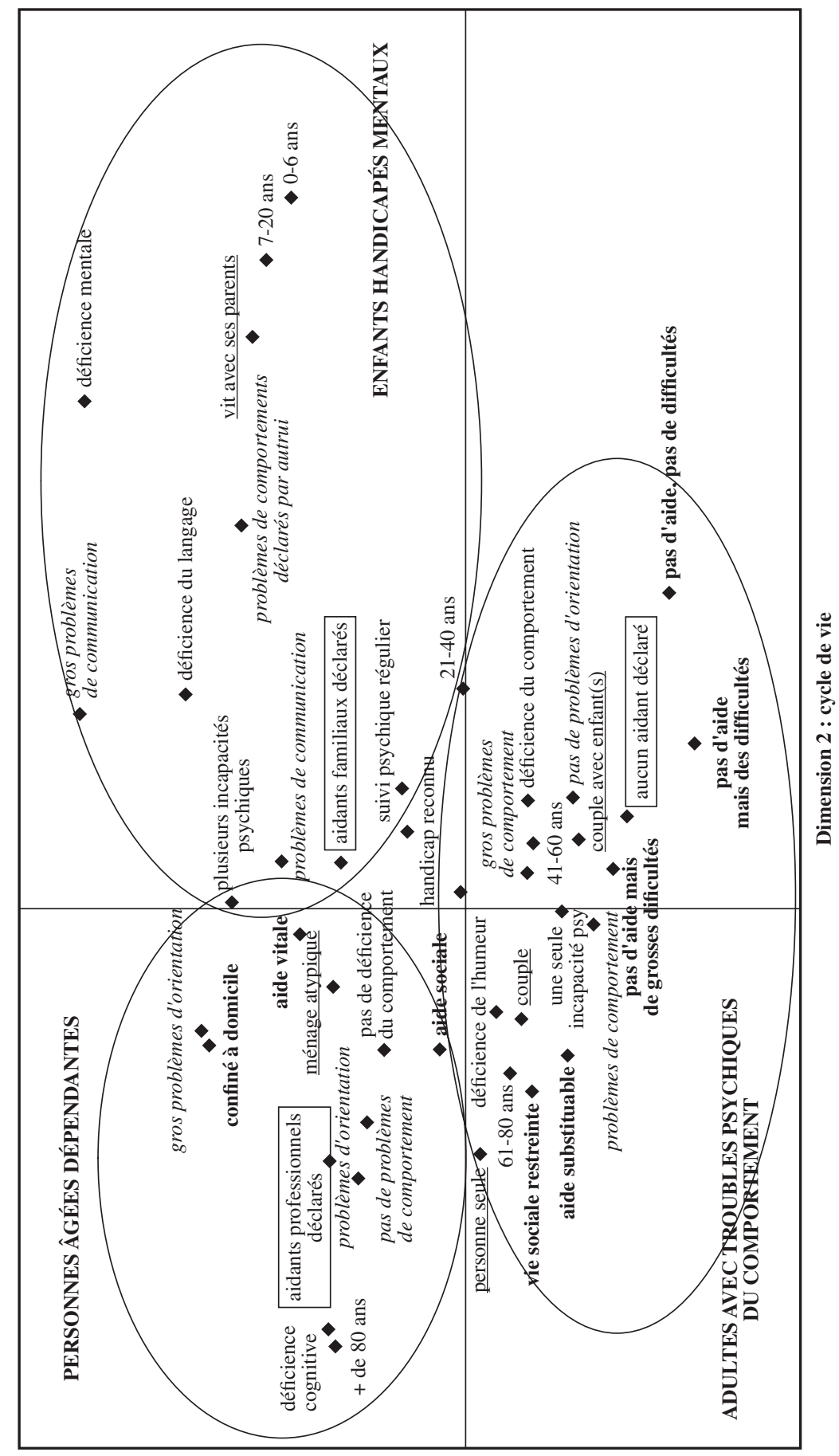

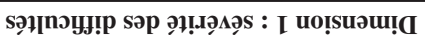


L'interprétation du premier axe ne pose guère de difficultés : il oppose clairement, au sud, les individus rencontrant le moins de difficultés psychiques à ceux, au nord, qui sont à l'inverse dans les situations de handicap les plus lourdes. Le deuxième axe, de manière plus étonnante, est fortement structuré par l'âge des individus : à l'est les plus jeunes, à l'ouest les plus âgés.

Le croisement de ces deux axes fait ressortir des pôles intéressants, qui renvoient à des configurations particulières : au nord-est, on trouve à la fois les plus jeunes, ceux qui ont des problèmes de communication, ceux qui ont des problèmes de comportement déclarés par autrui et ceux qui ont des déficiences mentales (et aussi du langage). Ces diverses caractéristiques renvoient à la figure des enfants handicapés mentaux avec troubles du comportement et/ou de la communication, comme par exemple les enfants autistes. Les modalités de prise en charge qui sont le plus associées à ces situations sont l'intervention d'aidants exclusivement familiaux mais aussi le recours à un suivi psychique régulier.

Au nord-ouest, on trouve plutôt des personnes âgées, des situations où le niveau d'aide est élevé (confinement à domicile notamment), des ménages atypiques (qui résultent souvent d'une recohabitation d'une personne âgée avec ses enfants), des personnes qui ont des problèmes d'orientation et celles qui ont une déficience cognitive. Le profil qui vient ici à l'esprit est celui des personnes âgées dépendantes atteintes de dégénérescences cognitives (type maladie d'Alzheimer). Il est intéressant de constater une nouvelle fois (Eideliman et Gojard, 2008) que des aidants professionnels sont bien plus souvent déclarés dans ce type de configuration.

Au sud du graphique, on trouve enfin un grand nombre de modalités, qui mélangent des situations où les problèmes sont apparemment minimes («pas d'aide, pas de difficultés », «pas de problèmes d'orientation », « aucun aidant déclaré»), mais il est surprenant de retrouver, associées à ces situations où aucune aide humaine ne semble mobilisée, les personnes ayant des problèmes de comportement, y compris sévères. Certes, le fait d'avoir isolé les problèmes de comportement déclarés par autrui conduit à surreprésenter, parmi ceux qui déclarent eux-mêmes des problèmes de comportement, les personnes d'âge actif (pour lesquelles on répond moins souvent au questionnaire que pour les plus jeunes ou les plus âgés) et de ce fait les personnes vivant en couple. Mais il est tout de même fort intéressant de remarquer que ces personnes d'âge actif, vivant souvent en couple et ayant parfois d'importants problèmes de comportement (mais uniquement ces problèmes, comme le montre la modalité «une seule incapacité psychique», associée à ces situations) déclarent peu d'aidants et semblent se débrouiller au quotidien malgré leurs difficultés. En d'autres termes, on a là des personnes qui «s'arrangent» avec des problèmes psychiques, peut-être en s'appuyant largement sur un soutien non explicitement déclaré de leur conjoint, sans recourir à de quelconque dispositifs d'aide, parfois au prix de difficultés quotidiennes importantes. 
L'importance du critère de l'âge recouvre en fait ici bien d'autres dimensions qu'il peut avoir tendance à masquer. Au sein même du paysage éclaté des troubles d'ordre psychique, les catégories d'âge semblent apporter un certain ordre : aux plus jeunes les troubles psychiques les plus proches des déficiences mentales, c'est-à-dire de retard d'intelligence; aux plus âgés les troubles psychiques les plus proches des déficiences cognitives, c'est-à-dire les conséquences psychiques des dégénérescences liées à des maladies du grand âge; aux âges intermédiaires enfin les troubles psychiques les plus classiques, caractérisés par des problèmes de comportement et d'humeur. Cette tripartition sommaire des troubles psychiques propose un tableau évidemment très simplifié de la nébuleuse des incapacités psychiques. Mais elle met bien en évidence combien les catégories diagnostiques et les systèmes politiques de prise en charge des troubles psychiques ont associé certaines pathologies à certaines étapes du cycle de vie. D'un point de vue médical, on peut avoir l'impression que ce sont ces étapes qui sont directement productrices de pathologies spécifiques : les limitations intellectuelles sont souvent présentes dès la naissance alors que les maladies mentales se déclarent généralement à l'adolescence ou à l'âge adulte; les dépressions sont plus fréquentes chez les personnes d'âge actif et peuvent être en partie rapportées aux contraintes, notamment professionnelles, liées à cet âge; les dégénérescences cognitives sont un produit possible du vieillissement biologique (Fassin et al., 2000). Mais une approche sociologique plus large, prenant en compte non seulement les processus pathologiques tels que les abordent les professionnels de la santé, mais aussi les formes de catégorisation des âges et des maladies, permet de questionner cette adéquation entre classes d'âges et classes de pathologies. Pourquoi les déficiences mentales n'apparaissent-elles plus aux âges actifs? Que deviennent les personnes atteintes de troubles psychiques une fois devenues âgées? Pourquoi ne pense-t-on que très rarement les dégénérescences cognitives en termes psychiques? Les frontières entre le mental, le cognitif et le psychique sont poreuses ${ }^{1}$ et leur solidité apparente est le fruit d'une construction des catégories médicales qui passe entre autres par le cycle de vie, c'est-à-dire par la manière dont les différents âges sont socialement construits. Bien sûr, les exceptions à la règle sont nombreuses et le graphique 4 montre d'ailleurs que d'autres caractéristiques rapprochent des personnes d'âges divers, même si l'importance du cycle de vie s'y fait encore fortement sentir.

1. Voir sur ce point l'article d'Aude Béliard et Jean-Sébastien Eideliman présent dans ce même numéro. 
Graphique 4 : Handicaps psychiques et arrangements pratiques - Axes 1 et 3

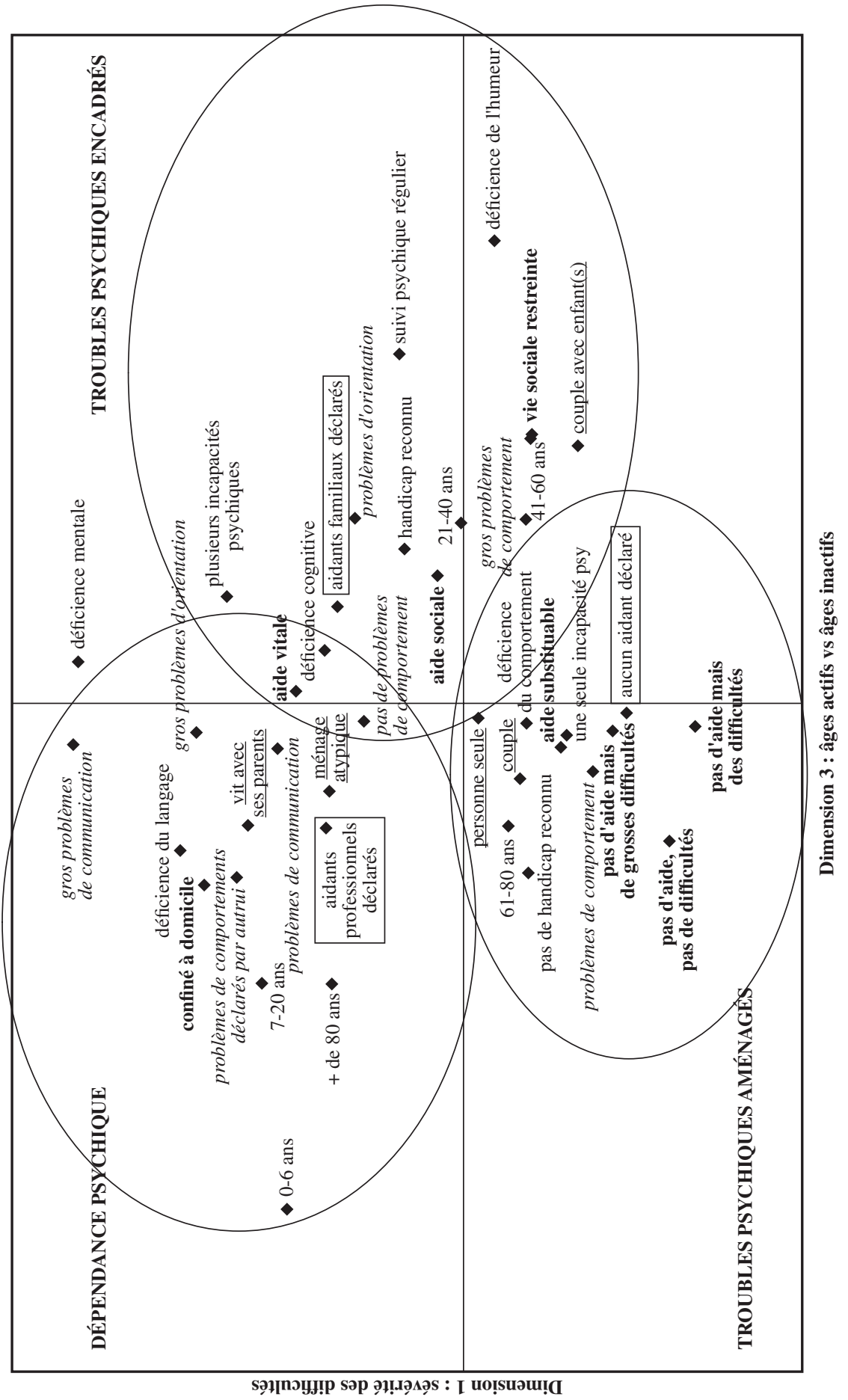


Le troisième axe ${ }^{1}$ retenu dans cette analyse des correspondances multiples semble lui aussi à première vue construit sur un pur critère d'âge : à l'ouest les plus jeunes et les plus âgés; à l'est les personnes d'âge intermédiaire; au centre (légèrement à l'ouest tout de même), les adultes vieillissants âgés de 60 à 80 ans. La distribution des caractéristiques sociales (comme la structure du ménage), des processus pathologiques et du réseau éventuel de soutien est pourtant bien différente de celle qui apparaissait sur le graphique 3 . Au nord-ouest du graphique, on trouve des individus qui ont la caractéristique commune d'être dans des situations de dépendance, comme le souligne leur statut au sein de leurs ménages : ils ne sont pas la «personne de référence», mais son enfant ou son parent. Leurs problèmes (de comportement notamment, mais aussi de communication) sont souvent déclarés par autrui et leur prise en charge souvent officialisée par la présence régulière à leurs côtés d'aidants professionnels, en association le plus souvent avec des aidants familiaux. On a donc là des situations où la dépendance est mise en avant, même si ce terme est habituellement réservé aux personnes âgées.

Au sud, on reconnaît l'essentiel des caractéristiques de ceux que nous avons précédemment appelés des adultes avec troubles psychiques du comportement : aucun aidant déclaré, des personnes vivant fréquemment en couple, des difficultés plus ou moins légères qui portent surtout, et uniquement, sur le plan du comportement. Ce sont cependant les adultes vieillissants qui sont ici les plus nombreux à être associés à ces caractéristiques. S'il fallait ne retenir qu'une expression, on pourrait dire qu'on a ici affaire à des troubles psychiques aménagés, au sens où ces personnes font avec des difficultés quotidiennes en s'appuyant, on peut le supposer, sur leur conjoint ou d'autres personnes de leur entourage qui les aident pour des activités dites substituables ${ }^{2}$. Ces troubles n'ont pas donné lieu à la mise en place d'un réseau d'aide spécifique, mais sont plutôt contenus, aménagés grâce aux routines mises en place petit à petit dans le cadre de l'organisation du ménage (ou d'un groupe d'entraide plus vaste).

Les adultes plus jeunes, c'est-à-dire les individus âgés de 20 à 60 ans, souvent en couple avec enfants, se trouvent en revanche tout à l'est du graphique et semblent dans une configuration tout à fait différente. De ce côté de l'espace social, on trouve des individus déclarant plusieurs incapacités psychiques, notamment des problèmes de comportement et des problèmes d'orientation. Les individus ayant une déficience de l'humeur (se traduisant le plus souvent par une dépression) y sont aussi surreprésentés. Contrairement à ce qui se passe pour les troubles psychiques aménagés, on

1. L'importance de ce troisième axe est moindre par rapport aux deux premiers car son pouvoir explicatif (mesuré par le pourcentage d'inertie du nuage de points qu'il explique; voir les résultats détaillés en annexe) est plus faible. Nous le commentons néanmoins pour affiner et nuancer les résultats des deux premiers axes.

2. Au sein de l'indicateur de niveau d'aide, la modalité « aide substituable» renvoie à des personnes qui déclarent une aide humaine pour des activités dont elles pourraient se passer en faisant appel à des services marchands non spécifiquement dédiés à des personnes handicapées ou dépendantes. 
est ici face à des troubles qui donnent lieu à des prises en charge spécifiques puisque des aidants familiaux sont souvent déclarés, de même que la mise en place d'un suivi psychique régulier, voire la reconnaissance officielle d'un handicap. L'aide reçue l'est le plus souvent pour des activités dites sociales, même si les cas d'abandon de certaines activités au détriment de l'intensité de la vie sociale ne sont pas rares. Nous proposons de parler de troubles psychiques encadrés, dans la mesure où le dispositif d'aide qui les entoure est bien visible, même si le recours à des aidants professionnels au sens strict (en dehors du suivi psychique) est rare.

L'analyse du troisième axe éclaire donc, à travers les deux derniers pôles développés, un autre pan des troubles psychiques, une autre distribution des configurations possibles. Il invite ainsi à ne pas trop figer les interprétations et à ne pas se contenter, en l'occurrence, d'une analyse des adéquations entre classes d'âges et classes de pathologies. Certes, chaque âge a ses catégories psychiques de référence, mais les décalages, combinaisons et spécificités apparaissent nombreux dès que l'on entre un tant soit peu dans le détail.

\section{口 Conclusion}

Ces analyses ne visent pas à dire de manière définitive qui sont les personnes ayant des handicaps psychiques, encore moins à définir ce qu'est le handicap psychique, mais plutôt à stimuler la réflexion sur ces questions en montrant que les définitions varient fortement selon le point de vue que l'on adopte. L'approche par les arrangements pratiques conduit à porter un regard critique sur une perception trop médicale ou administrative du problème : en partant d'indicateurs comme l'existence d'un suivi psychique régulier, la détermination d'une déficience par le corps médical ou encore la reconnaissance administrative d'un handicap, on se condamne à ne prendre en compte que les troubles les plus «encadrés » et à oublier les personnes qui font face à des difficultés quotidiennes en ne s'appuyant que sur leur expérience de ces difficultés et sur leur entourage. Certains types de troubles, comme la dépression, ont connu un succès social et médiatique (Ehrenberg, 1998) dont on peut penser qu'il facilite le recours à des formes d'encadrement de ces troubles (suivi psychique, reconnaissance administrative). Pour d'autres types de troubles, moins construits comme des entités homogènes dans l'esprit de la majorité, peut-être plus déstabilisants au quotidien car se situant toujours à la frontière du normal et du pathologique, l'encadrement est sûrement moins spontanément recherché, à moins qu'un événement particulier (crise aiguë nécessitant une hospitalisation, rencontre d'un professionnel posant un diagnostic ferme) ne vienne changer la manière de qualifier ce qu'on hésitait à voir comme des troubles psychiques (Moreau, 2007).

Par sa construction mettant l'accent aussi bien sur les déficiences que sur les incapacités, l'enquête Handicaps-Incapacités-Dépendance est le candidat idéal pour développer une approche par les arrangements pratiques, 
qui consiste à partir des difficultés quotidiennes exprimées pour ensuite saisir des situations dans toute leur dimension sociale. Au lieu de transformer en besoin d'aide insatisfait toute situation où des difficultés exprimées ne donnent pas lieu à la mobilisation d'un dispositif de soutien, cette approche vise à mettre au jour la diversité des façons de vivre avec des troubles psychiques, sans préjuger de la satisfaction ou de l'insatisfaction à être dans telle situation. Certes, on ne peut prétendre saisir toute l'ampleur des troubles psychiques à partir de quelques questions portant sur des problèmes de communication, d'orientation et de comportement, d'autant que l'on est dans le cadre d'une enquête autodéclarative; mais l'exploration de ces quelques difficultés permet déjà de mettre en lumière la grande diversité des manières de vivre avec un handicap psychique, dont l'existence d'un suivi psychique régulier est un bien pauvre indicateur.

L'accent mis sur la diversité des situations est aussi un bon remède à la vision misérabiliste de l'ensemble des personnes handicapées psychiques, qui sont souvent pensées comme des personnes souffrantes et isolées ${ }^{1}$. Si les troubles psychiques peuvent effectivement entraîner des ruptures et de la souffrance, il est très réducteur de les penser uniquement sous cet angle. L'analyse factorielle présentée dans cet article montre combien la diversité des configurations au sein des ménages est importante pour comprendre les différents arrangements pratiques qui sont mis en place. Enfermer les troubles psychiques dans une spirale de souffrance et d'isolement, c'est aussi contribuer à leur marginalisation en renforçant les peurs et les fantasmes qui les entourent et en rétrécissant de manière drastique le champ des problèmes concernés. L'approche statistique est un excellent remède à la myopie que nourrit la vision misérabiliste des troubles psychiques : elle permet de prendre de la hauteur, de «s'extraire des rues étroites pour monter au beffroi » (Héran, 2006) et par là d'accéder à une vue plus large et moins partiale de phénomènes terriblement complexes.

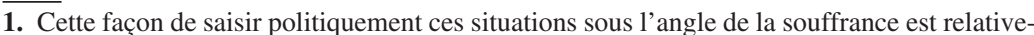
ment nouvelle et s'inscrit dans un mouvement plus général bien analysé par Didier Fassin (2002). 


\section{Bibliographie}

ANGUIS M., CHAPIREAU F., DE PERETTI C. (2003), «Les personnes suivies régulièrement pour troubles psychiques ou mentaux», Études et Résultats, DREES, $\mathrm{n}^{\circ} 231$, avril.

BESSIÈRE C. (2004), «Les "arrangements de famille". Équité et transmission d'une exploitation familiale viticole», Sociétés contemporaines, ${ }^{\circ}$ 56, vol. 4, p. 69-89.

BILAND É., EIDELIMAN J.-S., GOJARD S. (2008), «Ceteris (non) paribus? Combiner régression logistique et analyse des données pour étudier les arrangements pratiques des personnes handicapées ou dépendantes », Genèses, no 73, décembre, p. 37-56.

BLANC A., STIKER H.-J. (1998), L'insertion des personnes handicapées en France. Bilan et avenir d'un demi-siècle d'expérience, Paris, Desclée de Brouwer.

CASES C., SALINES E. (2004), «Statistiques en psychiatrie en France : données de cadrage », Revue française des affaires sociales, $\mathrm{n}^{\circ}$ 1, janvier-mars, p. 181-204.

CHAPIREAU F. (2003), «Connaissances nouvelles à propos des personnes recevant des soins de santé mentale », Revue française des affaires sociales, $\mathrm{n}^{\circ} 1-2$, janvier-juin.

CHAPIREAU F. (2004), «Le devenir sur deux ans des personnes hospitalisées en établissement psychiatrique», Études et Résultats, DREES, n 304, avril.

CHARZAT M. (2002), Pour mieux identifier les difficultés des personnes en situation de handicap du fait de troubles psychiques et les moyens d'améliorer leur vie et celle de leurs proches, Rapport à la ministre déléguée à la Famille, à l'Enfance et aux Personnes handicapées, Paris, Ministère de l'Emploi et de la Solidarité.

EHRENBERG A. (1998), La Fatigue d'être soi. Dépression et société, Paris, Odile Jacob.

EHRENBERG A., LOVELL A. (2001), «Pourquoi avons-nous besoin d'une réflexion sur la psychiatrie?», in Ehrenberg A., Lovell A. (dir.), La Maladie mentale en mutation. Psychiatrie et société, Paris, Odile Jacob, p. 9-39.

EIDELIMAN J.-S., GOJARD S. (2008), «La vie quotidienne à domicile des personnes handicapées ou dépendantes : du "besoin d'aide" aux arrangements pratiques », Retraite et Société, no 53 , janvier, p. 89-111.

FASSIN D. (2002), «La souffrance du monde. Considérations anthropologiques sur les politiques contemporaines de la compassion », L'Évolution psychiatrique, vol. 67, n ${ }^{\circ} 4$, p. 676-689.

HERAN F. (2006), Le Temps des immigrés. Essai sur le destin de la population française, Paris, Seuil.

LECLERC A., FASSIN D., GRANDJEAN H., KAMINSKI M., LANG T. (2000), Les inégalités sociales de santé, Paris, Inserm-La Découverte.

LETOURMY A. (1998), «La préparation d'une enquête sur les handicaps », Revue française des affaires sociales, ${ }^{\circ} 1$, janvier-mars, p. 27-33.

LIBERMANN R. (1988), Handicap et maladie mentale, Paris, PUF, coll. «Que sais-je ?». MOREAU D. (2007), Faire interner un proche? Le travail sur l'autonomie en contexte de troubles psychiques, Paris, CNAF, Dossiers d'études, nº 94, juillet.

PENNEC S. (2005), «Entre règles publiques et arrangements privés : le travail filial et la préservation des biens de famille», Enfances, Familles, Générations, n ${ }^{\circ} 2$.

RAVAUD J.-F., LETOURMY A., VILLE I. (2002), «Les méthodes de délimitation de la population handicapée : l'approche de l'enquête de l'INSEE "Vie quotidienne et santé" ", Population, $\mathrm{n}^{\circ}$ 57, p. 541-566. 
Vivre avec un handicap psychique à domicile : une approche statistique par les arrangements pratiques

SERAPHIN G. (dir.) (2003), Le handicap psychique chez les personnes majeures protégées : définition et indicateurs pour une recherche contextualisée, Paris, Institut Montaigne.

VELPRY L. (2008), Le Quotidien de la psychiatrie. Sociologie de la maladie mentale, Paris, Armand Colin.

WEBER F., GOJARD S., GRAMAIN A. (dir.) (2003), Charges de famille. Parenté et dépendance dans la France contemporaine, Paris, La Découverte.

WILSON M. (1993), «DSM-III and the Transformations of American Psychiatry: a History », American Journal of Psychiatry, vol. 150, n 3 mars, p. 399-410. 